(Aus dem physiologischen Institut der Universität Bonn.)

\title{
Lässt sich durch genügend lange Extraction mit siedendem Wasser sämmtliches in den Organen vorhandenes Glykogen gewinnen?
}

Von

Joseph Nerking.

Bei der Fortsetzung meiner Studien über die Art des Vorkommens des Glykogens in den Organen habe ich mir zunächst die Aufgabe gestellt, zu erforschen, ob es durch blosse Auskochung mit Wasser, wenn diese nur genügend lange fortgesetzt wird, möglich sei, sämmtliches in dem Organ vorhandenes Glykogen zu erhalten. Brücke hat bekanntlich zuerst auf diese Weise das Glykogen zu bestimmen gesucht, und es sind eine ganze Reihe principiell wichtiger Untersuchungen, insbesondere in Bezug auf die Entstehung des Glykogens im Thierkörper, nach dieser älteren Methode ausgeführt worden. Wenn das Glykogen in den Organen nur in freiem $\mathrm{Zu}$ stande vorhanden ist, so darf man bei der leichten Löslichkeit des Glykogens in heissem Wasser wohl annehmen, dass man es bei lange genug fortgesetzter Extraction vollständig gewinnen kann; ist es aber zum Theil wenigstens, was ich durch meine letzte Untersuchung ${ }^{1}$ ) für bewiesen erachte, chemisch gebunden, so wird man, bei einem bestimmten Punkte angelangt, durch Wasserextraction kein Glykogen mehr erhalten und zur Aufschliessung des ausgekochten Organpulvers schreiten müssen, um den Rest zu gewinnen. Von verschiedenen Autoren ist nun allerdings schon früher nachgewiesen worden, dass eine Extraction mit siedendem Wasser zur Gewinnung der Gesammtmenge des Glykogens nicht genügt; ob aber das Auskochen mit Wasser wirklich so lange fortgesetzt wurde, bis man absolut kein Glykogen mehr erhielt, ist zweifelhaft; meist wurde das Auskochen nur so lange fortgesetzt, bis der Auszug keine Opalescenz

1) Dieses Archiv Bd. 81 S. 8 ff. 
mehr zeigte, was auf absolute Glykogenfreiheit desselben durchaus noch nicht schliessen lässt. $\mathrm{R}$. $\mathrm{Kül \textrm {z } ^ { 1 }}$ ) bestimmt den nach der Wasserextraction in der Leber und den Muskeln zurückbleibenden Theil von Glykogen zu 20-25\% der Gesammtmenge. Weiter führt $\mathrm{K} u ̈ l \mathrm{z}$ an, dass bei Lebern mit hohem Glykogengehalte dieselben Werthe erhalten werden, mag man die Leber erst mit Wasser auskochen und dann mit Kali behandeln oder die Aufsehliessung direct vornehmen, während bei Lebern mit geringem Glykogengehalte die nach dem Kaliverfahren ausgeführten Bestimmungen immer günstiger ausfielen.

Neuerdings hat Austin ${ }^{2}$ ) darauf aufmerksam gemacht, dass aus Kalbsleber durch einfaches Auskochen im besten Falle nur ein Drittel des Glykogens zu erhalten ist; die von Austin angewandte Kochdauer ist jedoch sicher zu kurz gewesen.

$\mathrm{Zu}$ meinen eigenen Versuchen diente mir Kalbsleber. Ich habe die Leber zerkleinert, durch die Hackmaschine getrieben und die ganze Menge mit siedendem Wasser extrahirt. Die Extraction habe ich so lange fortgesetzt, bis ich in dem Auszug nach Anwendung der Brücke-Külz'schen Methode selbst nach mehrtägigem Stehen keine Spur von Trübung mehr erhielt. Dann erst habe ich den ausgepressten und getrockneten Rückstand, der zum Ueberfluss nochmals, mit negativem Erfolg, ausgekocht wurde, mit Kali behandelt. Ich babe ferner vor jeder Extraction, um dem Wasser immer neue Oberflächen zu bieten, den ausgekochten Brei in der Reibschale sorgfältig und längere Zeit durchgerieben.

\section{Versuch I.}

$745 \mathrm{~g}$ Kalbsleber wurden mit $1000 \mathrm{ccm}$ Wasser auf dem Wasserbade 24 Stunden lang ausgekocht. Darnach wurde abfiltrirt, ausgepresst, der Pressrückstand wurde wiederum mit $1000 \mathrm{ccm}$ Wasser 24 Stunden ausgekocht. Dieses Verfahren habe ich 18 Mal wiederholt, immer den Rüekstand vor jeder Extraction auf's Neue pulverisirend. Der 18. Auszug ergab nach Abscheidung der Eiweissstoffe mit Brücke's Reagens - die übrigens nur noch sehr gering war und Versetzen mit dem doppelten Volumen 96\% Alkohol keine Spur von Trübung mebr, selbst nach dreitägigem Stehen nicht.

1) Zeitschr. f. Biologie Bd. 22 S. $161 \mathrm{ff}$.

2) Archiv f. pathol. Anatomie und Physiologie Bd. 150 S. $185 \mathrm{ff}$. 
Trotzdem habe ich das ausgepresste und auf dem Wasserbade getrocknete Pulver nochmals mit $1000 \mathrm{ccm}$ Wasser in der Porcellanschale über freier Flamme $8 / 4$ Stunden ausgekocht mit vollkommen negativem Erfolg. Darnach wurde das Pulver auf dem Wasserbade getrocknet, das Gewicht desselben betrug nach dem Trocknen $110 \mathrm{~g}$. $25,8 \mathrm{~g}$ dieses trockenen Pulvers habe ich dann mit $200 \mathrm{ccm}$ Kalilauge von $2 \%$ und $200 \mathrm{ccm}$ Wasser im Wasserbade bis zur Lösung erhitzt, was 2 Stunden in Anspruch nahm. Die alkalische Lösung wurde nach dem Erkalten aufgefüllt auf $500 \mathrm{ccm}$, filtrirt und $100 \mathrm{ccm}$ Filtrat unter Zusatz von $3,1 \mathrm{cem} \mathrm{KOH}$ von $70,5 \%$ und $10 \mathrm{~g} \mathrm{JK}$ mit $50 \mathrm{ccm}$ Alkohol gefällt.

Die wässerigen Auszüge wurden vereinigt, auf etwa 2 Liter eingedampft, nach dem Erkalten auf 2 Liter aufgefüllt und $100 \mathrm{~cm}$ nach Zusatz von $4,2 \mathrm{cem} \mathrm{KOH}$ und $10 \mathrm{~g}$ JK mit $52 \mathrm{ccm}$ Alkohol von $96 \%$ gefällt. Das gefällte, mit alkoholisch-alkalischer Jodkaliumlösung und $66 \%$ Alkohol gewaschene Glykogen wurde invertirt in $300 \mathrm{cem} \mathrm{HCl}$ von 2,2\%. Je $81,2 \mathrm{~cm}$ dieser Lösung ergaben in:

Analyse I

$0,2020 \mathrm{~g} \mathrm{Cu}_{2} \mathrm{O}$
Analyse II

$$
0,2021 \mathrm{~g} \mathrm{Cu}_{2} \mathrm{O}
$$

$=0,0837 \mathrm{~g}$ Traubenzucker, in $300 \mathrm{cem}$ also $0,3092 \mathrm{~g}=0,2783 \mathrm{~g}$ Glykogen in $100 \mathrm{ccm}$ der wässerigen Lösung, in 2000 also: $5,566 \mathrm{~g}$ Glykogen in $745 \mathrm{~g}$ Leber. Die Leber enthielt demnach:

$0,7471 \%$ durch Wasser extrahirbares Glykogen.

Das aus der alkalischen Lösung gefällte und gewaschene Glykogen wurde invertirt in $200 \mathrm{~cm} \mathrm{HCl} \mathrm{2,2 \% .} \mathrm{Je} 81,2 \mathrm{ccm}$ ergaben in:

$$
\begin{array}{cc}
\text { Analyse I } & \text { Analyse II } \\
0,1005 \mathrm{~g} \mathrm{Cu}_{2} \mathrm{O} & 0,1003 \mathrm{~g} \mathrm{Cu}_{2} \mathrm{O}
\end{array}
$$

$=0,0393 \mathrm{~g}$ Traubenzucker in $81,2 \mathrm{~cm}$, in $200 \mathrm{ccm}$ also $0,0968 \mathrm{~g}$ entsprechend $0,0871 \mathrm{~g}$ Glykogen in $100 \mathrm{ccm}$ alkalischer Lösung, in 500 demnach: $0,4356 \mathrm{~g}$ in $25,8 \mathrm{~g}$ trockenen Pulvers; folglich waren in den zurückbleibenden $110 \mathrm{~g}$ noch vorhanden $1,8572 \mathrm{~g}$. In den $745 \mathrm{~g}$ verarbeiteter Leber waren also vorhanden insgesammt:

$$
0,9978 \%
$$

wovon $0,2492 \%=24,9 \%$ der Gesammtmenge nicht durch Wasser ausziehbar waren.

\section{Versuch II.}

$711 \mathrm{~g}$ Kalbsleber wurden mit $1000 \mathrm{ccm}$ Wasser 24 Stunden ausgekocht; darnach wurde ausgepresst, der Pressrückstand wurde 
im Mörser längere Zeit durchgerieben und wiederum mit $1000 \mathrm{ccm}$ Wasser 24 Stunden ausgekocht. Dieses Verfahren wurde $8 \mathrm{Mal}$ wiederholt. Der 8. Auszug gab, nach der Brücke-Külz'schen Methode geprüft, nur noch eine schwache Spur von Trübung beim Stehen; es wurde das Auskochen desshalb noch 2 Mal wiederholt, vor der letzten, 10. Auskochung war das feine Pulver getrocknet auf dem Wasserbad worden, in der Mühle gemahlen und gesiebt. Der letzte Wasserauszug gab auch nach mehrtägigem Stehen keine Spur von Trübung mehr nach Ausfällung der Eiweissstoffe mit Brücke's Reagens und Fällung des Filtrates mit Alkohol. Das von der Wasserauskochung zurückbleibende, getrocknete Pulver wog $105,5 \mathrm{~g}$. $25 \mathrm{~g}$ des trockenen Pulvers wurden mit $200 \mathrm{ccm} \mathrm{KOH}$ von $2 \%$ und 200 cem Wasser bis zur Lösung, 3 Stunden, erhitzt. Darnach wurde auf $500 \mathrm{ccm}$ aufgefüllt, filtrirt, $100 \mathrm{ccm}$ wurden nach Zusatz von $3,1 \mathrm{ccm} \mathrm{KOH}$ und $10 \mathrm{~g} \mathrm{JK}$ mit $50 \mathrm{cem}$ Alkohol $96 \%$ gefällt. Das filtrirte und gewaschene Glykogen wurde invertirt in $200 \mathrm{ccm} \mathrm{HCl}$ von 2,2\%. Je 81,2 ccm dieser Lösung ergaben in:

$\begin{array}{cc}\text { Analyse I } & \text { Analyse II } \\ 0,1485 \mathrm{~g} \mathrm{Cu}_{2} \mathrm{O} & 0,1485 \mathrm{~g} \mathrm{Cu}_{2} \mathrm{O}\end{array}$

$=0,0603 \mathrm{~g}$ Traubenzucker in $81,2 \mathrm{cem}$, in 200 demnach: $0,1485 \mathrm{~g}$ Traubenzucker entsprechend $0,13365 \mathrm{~g}$ Glykogen in $100 \mathrm{ccm}$ alkalischer Lösung, in 500 also $0,6683 \mathrm{~g}$ in $25 \mathrm{~g}$ trockenen Pulvers; es waren in dem Pulver nach der Wasserauskochung also noch vorhanden: $2,8202 \mathrm{~g}$

$=\mathbf{0 , 3 9 6 6} \%$ nicht durch Wasser extrahirbares Glykogen.

Weitere $25 \mathrm{~g}$ des trockenen Pulvers wurden mit $400 \mathrm{cem} \mathrm{KOH}$ von 10\% auf dem Drahtnetz erhitzt, Lösung war schon nach 5 Minuten eingetreten. Nach dem Erkalten wurde aufgefullt auf $500 \mathrm{ccm}, 100 \mathrm{cem}$ Filtrat wurden wie oben gefällt. Das filtrirte und gewaschene Glykogen wurde ebenfalls wieder in $200 \mathrm{ccm} \mathrm{HCl} \mathrm{2,2 \%}$ invertirt. Je 81,2 ecm ergaben in:

$\begin{array}{cc}\text { Analyse I } & \text { Analyse II } \\ 0,1595 \mathrm{~g} \mathrm{Cu}_{2} \mathrm{O} & 0,1593 \mathrm{~g} \mathrm{Cu}_{2} \mathrm{O}\end{array}$

$=0,0650 \mathrm{~g}$ Traubenzucker, in $200 \mathrm{ccm}$ also $0,1601 \mathrm{~g}$ entsprechend $0,1441 \mathrm{~g}$ Glykogen in $100 \mathrm{ccm}$ alkalischer Lösung, in $500 \mathrm{ccm}$ also $0,7205 \mathrm{~g}$; in den restirenden $105,5 \mathrm{~g}$ Fleischpulver waren noch vorhanden: $3,0405 \mathrm{~g}$ Glykogen

$=0,4276 \%$ nicht durch Wasser extrahirbares Glykogen. 
Die vereinigten wässerigen Auszüge der Leber wurden auf 1 Liter eingedampft, $100 \mathrm{cem}$ nach Zusatz von $4,2 \mathrm{ccm} \mathrm{KOH} \mathrm{von} 70,5 \%$ und $10 \mathrm{~g}$ JK mit $52 \mathrm{cem}$ Alkohol $96 \%$ gefällt. Das filtrirte und gewaschene Glykogen wurde invertirt in $300 \mathrm{ccm} \mathrm{HCl} 2,2 \%$. Je 81,2 cem ergaben in:

$$
\begin{array}{cc}
\text { Anaiyse I } & \text { Analyse II } \\
0,0750 \mathrm{~g} \mathrm{Cu}_{2} \mathrm{O} & 0,0750 \mathrm{~g} \mathrm{Cu}_{2} \mathrm{O}
\end{array}
$$

$=0,0282 \mathrm{~g}$ Traubenzucker, in $300 \mathrm{ccm}$ demnach $0,1042 \mathrm{~g}$ entsprechend $0,0938 \mathrm{~g}$ Glykogen in $100 \mathrm{ccm}$ Lösung, in $1000 \mathrm{ccm}$ $0,938 \mathrm{~g}$

$$
=0,1819 \% \text { durch Wasser extrahirbares Glykogen. }
$$

Bei dieser Leber sind also trotz anhaltender Extraction mit siedendem Wasser bis zur vollständigen Erschöpfung nur 24,9\% bezw. 23,6\% der Gesammtmenge des Glykogens in dem wässerigen Auszug enthalten gewesen, während 75,1 bezw. $76,4 \%$ erst durch Behandlung mit $\mathrm{KOH}$ zu erhalten waren.

Wie viel von der Gesammtmenge des Glykogens in den Muskeln durch Extraction mit siedendem Wasser nicht erhaltbar ist, habe ich noch nicht untersuchen können, hoffe darüber aber in meiner nächsten Veröffentlichung berichten zu können. Den Einwand, dass durch die Behandlung mit siedendem Wasser in Folge der Coagulation eine grössere Verdichtung des Eiweisses eintritt und das Glykogen mechanisch fest eingeschlossen wird, muss ich zurückweisen aus dem einfachen Grunde, weil ich, wie ich nomals ausdrücklich betone, vor jeder Extraction durch ernenertes Pulverisiren dem Wasser immer neue Oberflächen zum Angriff geboten habe. Bisher war man wohl geneigt, anzunehmen, dass man im Stande sei, durch Wasser das Glykogen allmälig, aber schliesslich doch vollständig auszuziehen; dies ist jedoch nicht zutreffend; es gibt eine Grenze, bei welcher selbst durch anhaltendste Extraction mit siedendem Wasser kein Glykogen mehr erhalten werden kann, und ich neige der Ansicht zu, dass, wie ich schon in meiner letzten Arbeit andeutete, nur das durch Wasser extrahirbare Glykogen in freiem Zustande in den Organen vorhanden, während der Rest chemisch gebunden ist. 
F

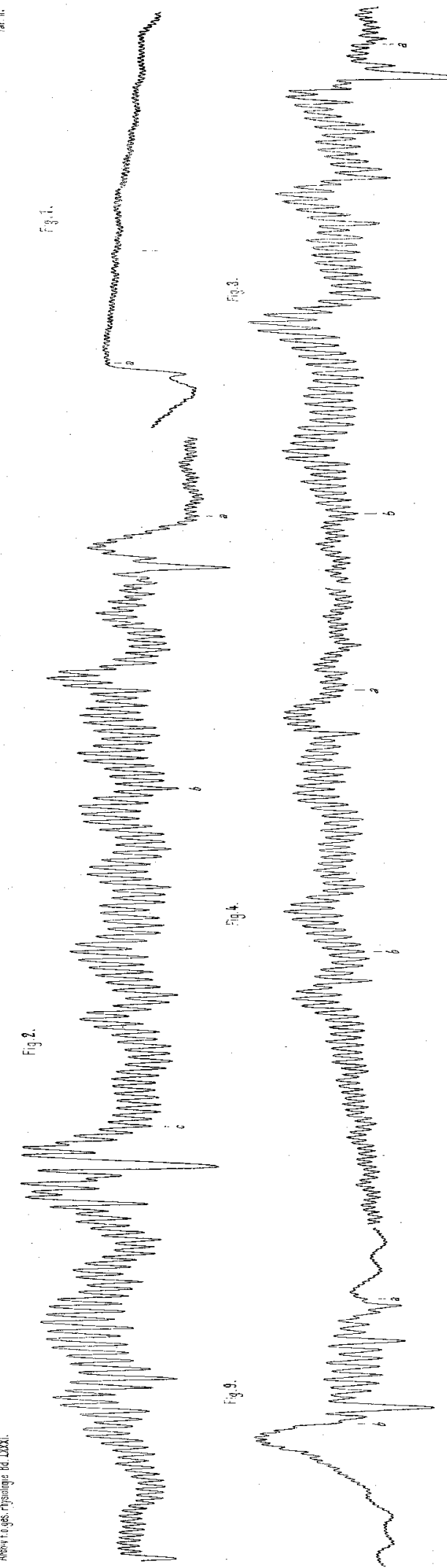

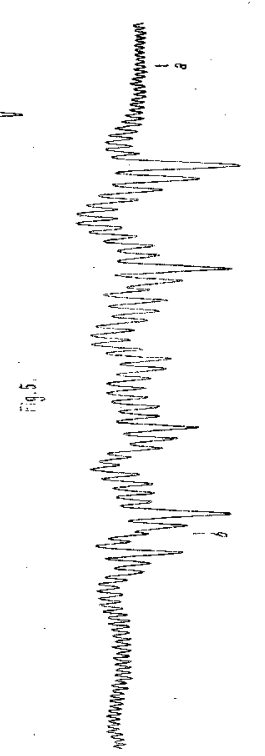
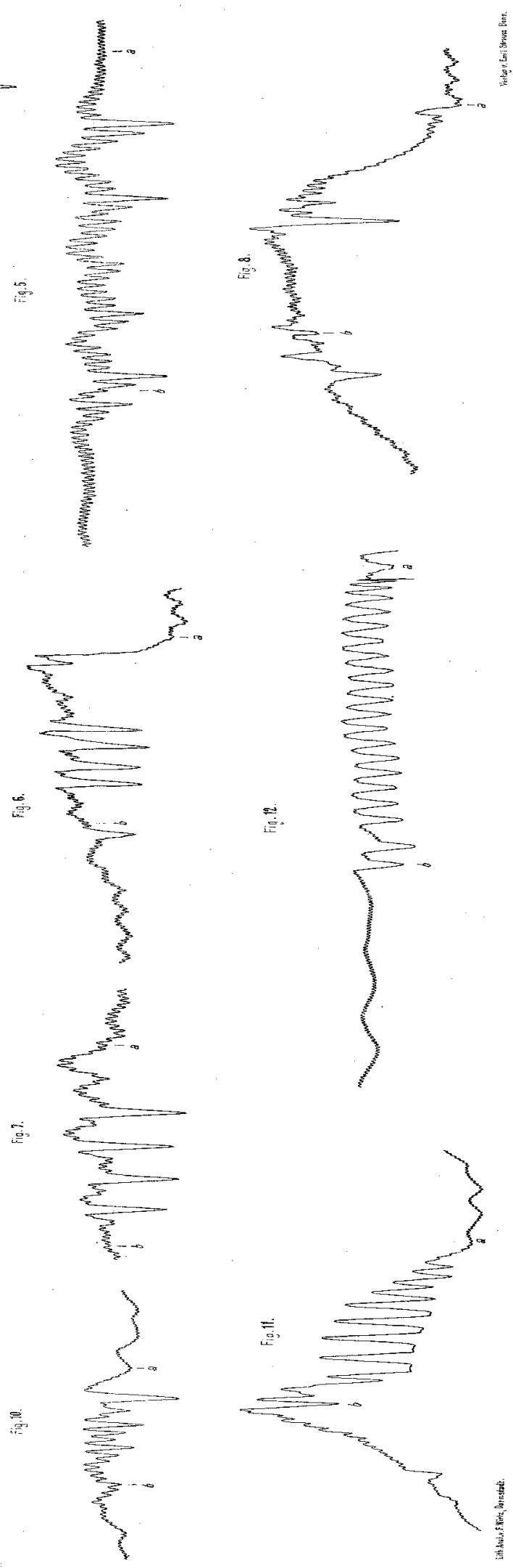


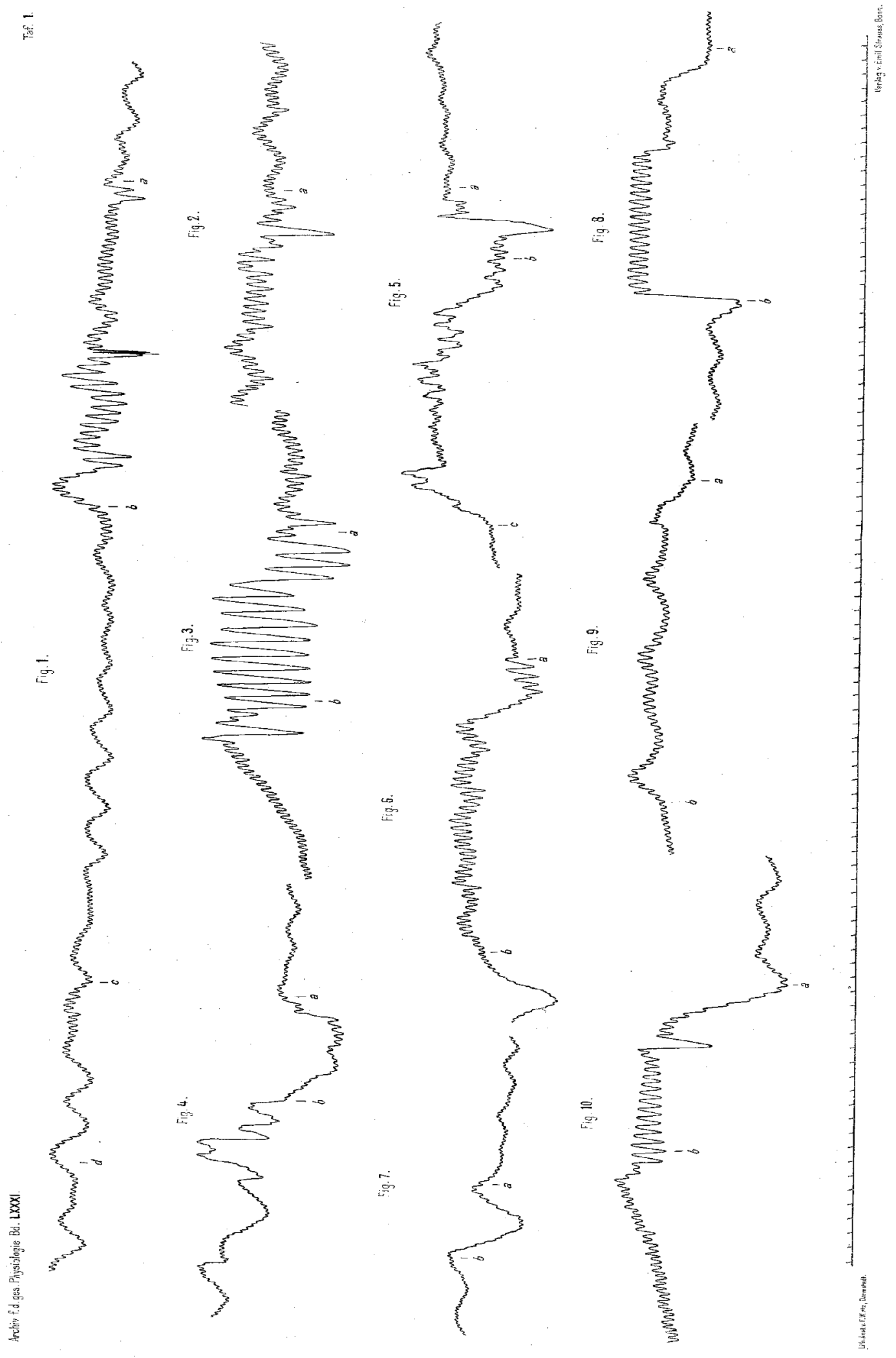



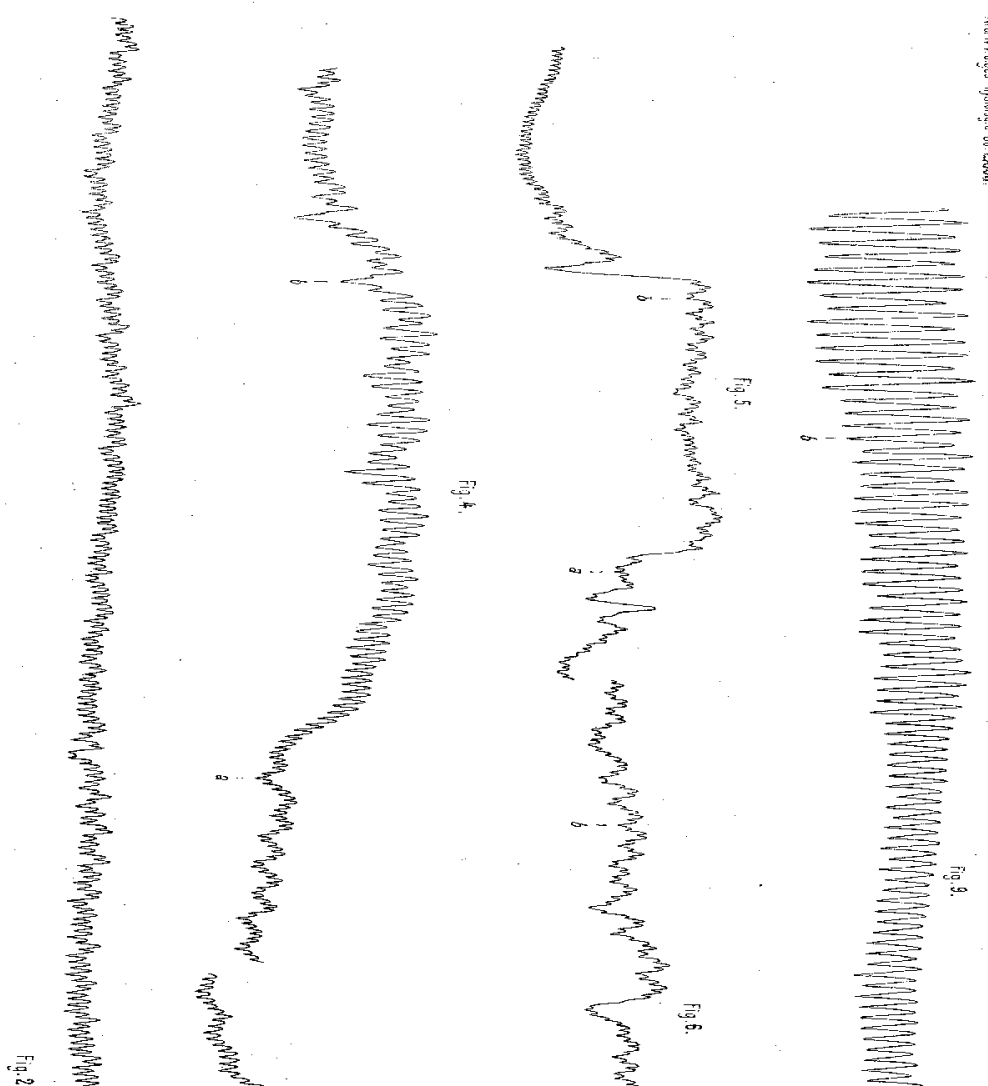

,
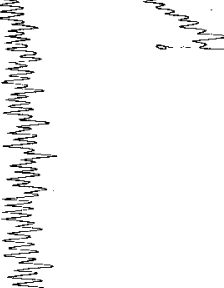

$\bar{P}^{72}$
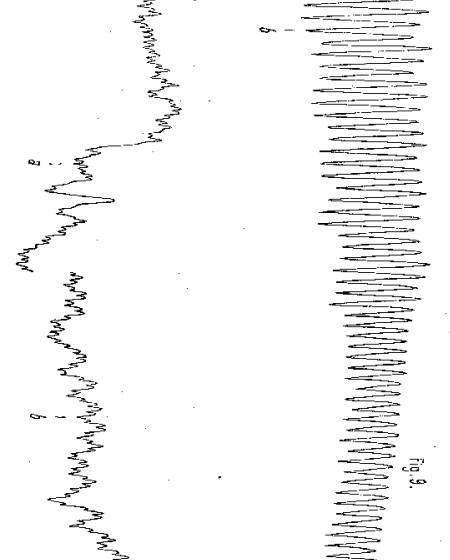

$=$

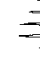

